\title{
PROBLEMATIQUES GENERALES
}

\section{La libéralisation de la filière cacaoyère ivoirienne et les recompositions du marché mondial du}

cacao : vers la fin des « pays producteurs » et du marché international ?

Oléagineux, Corps Gras, Lipides. Volume 8, Numéro 6, 566-76, Novembre - Décembre 2001, Dossier : L'avenir des cultures pérennes

Auteur(s) : Bruno LOSCH, Cirad, TA 60/15, 73, avenue J.-F.-Breton, 34398 Montpellier Cedex 5, France.

Résumé : La Côte d'Ivoire occupe une place privilégiée dans le monde du cacao puisqu'elle a conquis le premier rang mondial sur le Ghana en 1977. Ce leadership s'est surtout accompagné d'une croissance exceptionnelle de la production qui atteint entre 40 et $45 \%$ de l'offre mondiale depuis le milieu de la décennie 90 (de l'ordre de 1,2 million de tonnes) et presque $50 \%$ des échanges. Cette situation assez exceptionnelle parmi les marchés des soft commodities explique que la Côte d'Ivoire soit l'objet d'une grande attention de la part des différents acteurs du marché. Ainsi le processus de libéralisation de la filière cacao au cours des années 90 et le démantèlement de la toute puissante caisse de stabilisation (la CSSPPA, appelée couramment la "Caisse " ou "Caistab ») en 1999, tout comme la volonté du gouvernement ivoirien de remettre en place aujourd'hui (juillet 2001) un mécanisme de stabilisation sont à l'origine d'abondants commentaires de la presse spécialisée et d'autant de réactions des différents protagonistes du monde du cacao. Ces changements ont eu en effet, ou auront, d'importantes conséquences sur les conditions d'écoulement de la production, sur les conditions de l'accès à l'offre ivoirienne ainsi que sur la dynamique du marché international et l'évolution des prix. Simultanément, la place et le poids du secteur cacaoyer dans l'économie politique ivoirienne (à la fois dans les grands agrégats économiques et dans les positions de pouvoir des intérêts privés locaux) en ont fait un enjeu central des recompositions économiques et politiques de la dernière décennie. La grave crise politique de l'année 2000, qui a fait suite au premier coup d'État de l'histoire du pays (décembre 1999), n'est pas sans liens avec la libéralisation imposée de l'extérieur par les bailleurs de fonds multilatéraux $[1,2]$. Et l'évolution de la filière cacao reste aujourd'hui un point central des négociations entre le gouvernement et les agences d'aide. Cette libéralisation de la filière ivoirienne, qui correspond à un ajustement tardif aux nouvelles modalités de fonctionnement de l'économie mondiale, s'est accompagnée d'une perte progressive de contrôle des acteurs publics et privés locaux et d'une perte d'influence sur le marché. Cette évolution est en apparence paradoxale du fait de la place occupée par le pays dans l'offre mondiale, mais aussi à cause de l'évolution structurelle de la filière qui a été caractérisée par le développement rapide des capacités industrielles de broyage (presque triplées depuis 1995). Avec un potentiel de 300000 tonnes (et bientôt 350 000), la Côte d'Ivoire est en effet devenue le principal " broyeur à l'origine " du marché, c'est-à-dire un pays producteur assurant une première transformation de ses fèves alors que la plupart des autres pays ont dû limiter leurs ambitions industrielles du fait des handicaps du broyage sur les lieux de production. Ces différents phénomènes emboîtés - croissance de l'offre, croissance des broyages, libéralisation ont bien évidemment fortement influencé la physionomie de la filière : arrivée de nouveaux agents, développement de nouvelles fonctions, apparition de nouveaux modes de coordination. La compréhension de ces changements, en regard des évolutions globales du marché et de ses acteurs, nécessite d'adopter une lecture historique permettant de replacer dans leur contexte les stratégies 
mises en œuvre par les différents agents publics et privés, nationaux, étrangers et internationaux ; elle nécessite aussi de mobiliser des registres explicatifs qui relèvent aussi bien des enjeux internes propres à la Côte d'Ivoire que de ceux du marché mondial du cacao. Notre propos s'articulera en trois points. Nous rappellerons tout d'abord les conditions du développement de la filière ivoirienne, les raisons de sa forte croissance et les limites de son pouvoir de marché. Nous nous attacherons ensuite à l'analyse des stratégies mises en œuvre par les firmes nationales et étrangères à l'occasion de la libéralisation et plus particulièrement aux motifs et aux modalités des alliances nouées entre les différents types d'opérateurs, notamment en ce qui concerne le broyage. Nous examinerons enfin les nouvelles caractéristiques de la coordination entre les différents agents de la filière et leurs conséquences sur les conditions de l'approvisionnement en quantité et en qualité.

Summary : The restructuring of the world cocoa market has concluded with the liberalisation of the sector in the world's leading producing country - Côte d'Ivoire - clearing the way for domination by an oligopoly of global companies. This paper describes how Côte d'Ivoire's share of world production created an illusion but not the reality of market power. In the 1990s, in the wake of failed attempts to influence the world market, the Ivorian cocoa experienced a series of upheavals that were both pivotal to broader changes in the global market and a reflection of them. The converging strategies of new Ivorian firms and of the major global grinding companies resulted in increased vertical integration in Côte d'Ivoire, exemplified in the development of "origin grinding". Later, financial difficulties encountered by Ivorian firms led to global companies taking control. Amongst the results of these changes are a decline in the role of traders, a redefinition of relationship between grinders and chocolate manufacturers, and a standardisation of cocoa quality around an average "bulk" level. This signals the end of "the producing countries" and of the global market.

Keywords : cocoa, Côte d'Ivoire, liberalisation, vertical integration, global companies.

\section{ARTICLE}

\section{La conquête du premier rang mondial et ses illusions : des origines à la " guerre du cacao »}

\section{Rappels sur le développement et les caractéristiques de la filière}

L'introduction du cacao en Côte d'Ivoire date de la seconde moitié du xix siècle mais le développement de la filière n'a véritablement débuté qu'à partir des années 30 alors que le pays était une colonie française. Les bases de son régime de croissance se sont mises en place à compter des années 50 et sont à l'origine d'une évolution rapide de l'offre depuis l'indépendance en 1960 et surtout depuis les années 70, au rythme moyen de plus de $8 \%$ par an (de 0,2 à 1,2 million de tonnes entre 1970 et 1995).

La physionomie de la filière jusqu'à sa libéralisation au cours de la décennie 90 est le résultat d'une cinquantaine d'années de stabilité (1930-1980), aussi bien au niveau de son environnement international que dans les conditions de sa régulation interne. En effet, malgré de nombreuses évolutions dans le statut des acteurs et leur hiérarchie ou dans les modalités techniques de son fonctionnement, la filière a été marquée par l'importance des protections de marché liées au rôle des États-nations et au poids de l'intervention publique dans le fonctionnement des économies. Cette 
stabilité n'a pas été remise en cause par l'indépendance en 1960, du fait des choix politiques du nouvel État ivoirien qui a fait le pari de la continuité par le perfectionnement des institutions et l'approfondissement des alliances avec les firmes étrangères.

Au niveau du marché international, la Côte d'Ivoire a répondu à une demande croissante en volume et stable en qualité. Les industries utilisatrices d'Europe et d'Amérique du Nord étaient avant tout à la recherche d'un produit sain et homogène mais peu différencié, permettant de satisfaire les besoins d'une consommation en expansion liée à l'évolution générale du niveau de vie des pays industrialisés. Le marché cacaoyer - à l'instar d'autres marchés de matières premières - a tout d'abord été segmenté selon le principe de la préférence impériale qui offrait une protection douanière (chaque empire colonial approvisionnant sa métropole). II s'est ensuite globalisé dans le cadre d'une régulation internationale caractérisée par un oligopole d'États-nations (producteurs et consommateurs) s'efforçant de coopérer par des alliances ad hoc puis par un accord international, mis en place en 1972, après une longue gestation. L'offre cacaoyère était coordonnée par des dispositifs publics centralisés (boards, offices, caisses, instituts) qui étaient à l'origine de standards nationaux établis en référence à la qualité "Ghana ", alors premier producteur mondial, qui servait d'étalon de qualité pour la détermination des prix (et des décotes).

Au niveau de la production, l'offre ivoirienne se caractérise par un produit moyen non différencié proche du standard Ghana du fait de la similitude des conditions agro-climatiques et techniques - qui résulte des caractéristiques de la demande mondiale pour du cacao " tout-venant " et de I'homogénéité des systèmes de production (pratiques extensives et faible niveau technique liés à l'abondance en terre et en travail et à l'absence de capital). La croissance de l'offre a été favorisée par des politiques publiques qui garantissaient l'accès à la main-d'œuvre (grâce aux migrations) et au foncier (conditions d'installation libérales y compris pour les travailleurs migrants), mais qui assuraient aussi l'accès à un prix et à un achat du cacao garantis par le système de stabilisation. Le prix officiel était unique, quelle que soit la qualité du produit, renforçant ainsi la tendance à un produit de masse sans différenciation.

$\mathrm{Au}$ niveau de l'exportation, les sociétés de traite coloniales (CFAO, SCOA, CFCI) ont été progressivement remplacées au tournant de l'indépendance par des exportateurs spécialisés d'origine étrangère (les principaux étaient SIFCA, JAG, SHAC, DAFCI), qui ont peu à peu ouvert leur capital à des intérêts locaux ou qui ont participé à la création de joint ventures promues par les pouvoirs publics et rétrocédées ensuite à des capitaux privés (principalement la société Comafrique). À la différence des marketing boards qui ont évolué rapidement vers une exclusion des opérateurs privés, la création du système de stabilisation à l'époque coloniale (1955) s'est traduite par le maintien opérationnel des exportateurs privés, accompagné d'une gestion centralisée de l'offre ivoirienne par la Caisse. Ce dispositif a été maintenu après l'indépendance et les pouvoirs de la Caistab ont été progressivement accrus sans jamais remettre en cause l'existence des exportateurs dont l'activité était cependant réglementée par un système de quotas d'achat. Cette configuration s'est traduite par l'apparition d'exportateurs fictifs qui bénéficiaient de protections politiques, parallèlement aux véritables exportateurs qui prenaient en charge la gestion physique du produit et se livraient à une concurrence pour le contrôle du marché des quotas.

Au niveau de la commercialisation intérieure - appelée la " traite " -, la coordination entre agents (planteurs, collecteurs ou " pisteurs ", " traitants ", exportateurs) reposait sur des réseaux commerciaux fondés sur la confiance et la durée et sur un système d'avances monétaires remboursables (appelées mandats) octroyées par les exportateurs à partir de crédits bancaires. En 
revanche, le système coopératif est resté embryonnaire du fait des réticences et des obstacles du pouvoir politique. Cette coordination verticale a été consolidée et renforcée par la mise en place du dispositif de stabilisation, avec un système administré appelé " barème ", piloté par la Caistab, qui fixait annuellement les prix à chaque étape de la filière (bord champ, centre de groupage, magasin exportateur, magasin portuaire, $\mathrm{FOB}, \mathrm{CAF}$ ). Le traitement de la qualité du produit était pris en charge par les exportateurs (nettoyage, calibrage, séchage complémentaire) sous contrôle final de la caisse de stabilisation.

Au niveau de la transformation enfin, la Côte d'Ivoire a mis en place, comme nombre d'autres pays producteurs dès le début des années 60 , une industrie de broyage des fèves ${ }^{2}$. Ce choix répondait à une double logique :

- une logique commerciale propre à la filière cacao, qui était destinée à améliorer ses performances grâce à la valorisation par la transformation des fèves de mauvaise qualité, mais qui permettait, simultanément, de renforcer la qualité des exportations en produit brut ;

- une logique de promotion industrielle plus globale, qui correspondait aux objectifs de développement économique du gouvernement.

Trois sociétés industrielles de broyage ont été créées et ont bénéficié de participations publiques, de subventions et de régimes fiscaux privilégiés (sur les impôts et taxes d'exportation). La première (SACO) a été créée par un industriel français (Cacao Barry) en 1962 ; les deux autres (API et PROCACI) ont été constituées par l'État en 1968 et 1977 avec des contrats de gestion privés, puis rétrocédées respectivement à SACO et à une nouvelle firme ivoirienne créée pour l'occasion (UNICAO) ${ }^{3}$. Enfin, la Côte d'Ivoire possède également une petite industrie de seconde transformation avec la société CHOCODI, créée en 1974 par l'État et la SACO, qui produit de la masse et du chocolat de couverture destinés à l'exportation ainsi que des tablettes, de la poudre et de la pâte à tartiner destinés au marché local. Nestlé produit également de la poudre de petit déjeuner à partir de poudre fournie par CHOCODI ${ }^{4}$.

Ainsi, au milieu des années 80 , la configuration de la filière cacao ivoirienne était caractérisée par l'existence d'une production de masse à marché protégé, assurée par environ 600000 producteurs de taille petite à moyenne (entre 5 et 20 hectares) avec un système de commercialisation reposant sur environ 800 traitants, deux broyeurs, un chocolatier, une cinquantaine d'exportateurs et une caisse de stabilisation. Ce dispositif correspondait à un oligopsone lâche d'une dizaine d'exportateurs véritablement opérationnels pour les achats intérieurs et à un monopole public de la Caistab pour les exportations. Cet ensemble était consolidé par la force des alliances de classe renforcées et légitimées par le leadership d'Houphouët-Boigny, président de la République et père de l'indépendance. Ces alliances regroupaient, d'une part, le pouvoir politique et les firmes privées (sur la base des avantages accordés par l'État aux entreprises en échange de leur " compréhension » vis-à-vis de l'actionnariat politique) et, d'autre part, le pouvoir politique et les planteurs (sur la base de la sécurisation des revenus par les prix, de l'accès à la terre et au travail migrant en échange d'un soutien électoral). Elles sont à l'origine d'un complexe politico-économique original qui permet de comprendre la trajectoire suivie par la Côte d'Ivoire ${ }^{5}$. 


\section{L'impossible pouvoir de marché ivoirien}

La croissance de l'offre ivoirienne dans les années 70 et 80 , son poids dans l'économie nationale et son contrôle par l'État se sont traduits par une volonté de la Côte d'Ivoire d'acquérir un pouvoir de marché sur le marché international du cacao avec l'objectif d'influencer la formation du prix.

Pendant une première période, la Côte d'Ivoire s'est efforcée de participer à la construction d'un pouvoir de marché des pays producteurs par une politique de régulation de l'offre définie dans le cadre d'accords inter-États. Comme l'a bien montré Mac Calla [4], le pouvoir de marché sur les marchés agricoles ne peut pas se réduire à la seule part de marché. Du fait des spécificités de l'offre agricole et de ses rigidités - atomisation des producteurs, aléas climatiques et délais d'adaptation, en particulier pour les cultures pérennes dont l'entrée en production est différée (trois à quatre ans pour le cacao) -, le pouvoir de marché repose avant tout sur les stocks et leur maîtrise et donc sur la capacité de stockage. Les tentatives d'intervention des pays producteurs ont ainsi porté sur la création de stocks régulateurs au niveau international ou au niveau national par la mise en œuvre d'une stratégie d'offre résiduelle.

Ainsi, la Côte d'Ivoire a pris part à l'ensemble des consultations internationales qui, depuis 1956 sous l'égide de la FAO puis des Nations unies (Cnuced), aboutiront à l'Accord international de 1972 qui réunit producteurs et consommateurs et institue un système de contingentement et un stock international. Parallèlement, elle a participé à la fondation de l'Alliance des producteurs de cacao créée en 1962 à Abidjan à l'instigation du Ghana. L'Alliance n'obtiendra jamais de succès véritable et l'Accord fonctionnera très difficilement. Outre les problèmes classiques de l'action collective liés aux stratégies opportunistes des pays membres (en particulier le non-respect des règles ou le phénomène du « passager clandestin " ${ }^{6}$ ), l'Accord a buté de façon récurrente sur la difficulté du stockage. En effet, contrairement au café, le cacao se stocke difficilement et, surtout, il supporte mal l'entreposage dans les pays producteurs où le taux d'humidité du climat tropical ne permet pas de le maintenir plus de trois mois sans dommages. Cette caractéristique explique la faiblesse du stockage à l'origine et sa réalisation dans les pays tempérés consommateurs qui disposent ainsi d'un avantage stratégique certain. Les seules possibilités offertes aux pays producteurs sont :

- la réalisation d'entrepôts spécifiques et onéreux (par exemple en atmosphère contrôlée) ;

- la transformation locale (la conservation de la masse et du beurre étant plus aisée) ;

- ou l'acquisition de capacités de stockage dans les pays consommateurs, qui n'ont manifestement pas le même impact psychologique sur les acteurs du marché.

En réponse à la faible efficacité de l'Accord face à la baisse des cours de la fin des années 70, qui faisait suite au boom des prix de 1977 à 1979, la Côte d'Ivoire choisit de sortir de l'Accord en $1980^{7}$ et essaya de relancer I'Alliance. Elle s'engagea surtout dans une tentative d'action individuelle qui lui sera fatale en se laissant prendre par les illusions du pouvoir de son premier rang mondial. À plusieurs reprises, le pays s'est alors retiré du marché pendant plusieurs mois pour essayer de peser sur les cours. Parallèlement, il développait des capacités de stockage spécifiques de plus de 100000 tonnes entre 1979 et 1983, augmentait ses capacités de broyage de 45000 tonnes (1988) et, enfin, inaugurait la pratique des block deals en passant le premier " contrat du siècle " avec le négociant britannique Rayner (100 000 tonnes en 1980). La Côte d'Ivoire va poursuivre dans cette voie lors de l'effondrement 
des cours, à compter de 1986, en adoptant une stratégie de rupture totale : après avoir suspendu le remboursement de sa dette extérieure en mai 1987, elle engagea ce que l'histoire a retenu comme la " guerre du cacao » [6]. Entre juillet 1987 et octobre 1989 (27 mois), la Côte d'Ivoire tenta d'arrêter la chute des cours en bloquant toutes ses exportations et en essayant d'organiser des stockages en Europe sous couvert de block deals passés avec deux géants du négoce de l'époque ${ }^{8}$. L'échec fut total, puisque le marché attendait la sortie des fèves ivoiriennes, et les conséquences pour le pays furent désastreuses : implosion du système de prix garantis, non-paiement des producteurs, économie sinistrée et en banqueroute. Elles déboucheront sur une crise politique grave et sur une libéralisation de l'économie et de la filière cacao imposée par les bailleurs de fonds internationaux.

Cette impossible conquête d'un pouvoir de marché par la Côte d'Ivoire peut s'expliquer par quatre facteurs.

Premièrement, la Côte d'Ivoire, au moment de ses tentatives des années 80, n'est pas un acteur suffisamment dominant :

- elle ne détient qu'entre 20 et $30 \%$ de l'offre mondiale (en fin de période) ;

- son retrait du marché profite aux autres producteurs (notamment l'Indonésie et la Malaisie) ;

- les industries utilisatrices européennes et américaines détiennent les capacités de stockage d'un marché en surproduction.

Deuxièmement, le poids économique et politique du cacao au niveau national rendait non viable une stratégie de ce type : le pays était trop dépendant de sa filière cacao pour pouvoir résister longtemps ${ }^{9}$.

Troisièmement, le fonctionnement du marché international s'est profondément modifié : ce sont les marchés à terme qui sont désormais prépondérants et ils ont fortement réduit le poids stratégique du " cacao physique » en général et du cacao ivoirien en particulier ${ }^{10}$.

Enfin, quatrièmement, la montée en puissance rapide des grands groupes industriels mondiaux du broyage et de la chocolaterie leur permet dorénavant de prendre une place stratégique réelle dans le fonctionnement du marché au détriment des États et du négoce.

Ce nouveau rapport de force a plusieurs origines.

Il s'agit bien sûr, en premier lieu, du changement de régime de l'économie mondiale, lié à l'érosion des bases de la croissance des économies nationales, qui incite les firmes puis les États à s'engager dans la compétition internationale $[8,9]$. Ce besoin de compétition est le principal motif de la libéralisation, dont l'objectif est relayé par les bailleurs de fonds; et le désengagement des États débouche logiquement sur la fin des systèmes d'offre centralisés et sur le dépérissement des accords internationaux.

Il s'agit, ensuite, de la perte progressive de l'influence et de la place du négoce qui est la conséquence de l'amélioration des techniques de commercialisation, tant sur le plan de la gestion physique que sur celui de la couverture des risques : les progrès considérables des moyens de communication (information, transport maritime) diminuent les temps de négociation et d'acheminement et permettent des rotations beaucoup plus courtes, avec le développement d'une tendance aux flux tendus [10] ; et le recours aux marchés à terme permet de gérer les risques techniques et surtout financiers. La fonction traditionnelle des négociants, qui était de prendre en charge l'interface entre 
l'offre et la demande, s'en trouve considérablement réduite.

En dernier lieu, la baisse historique des cours à la fin de la décennie 80, qui n'est pas répercutée au niveau du consommateur final, a offert aux firmes une marge de manœuvre considérable pour leur redéploiement. Elle va être utilisée dans des opérations de rachats et de fusions à grande échelle qui vont se poursuivre jusqu'à aujourd'hui et bouleverser totalement la physionomie du marché du cacao.

L'oligopole historique d'États-nations de l'après-Seconde Guerre mondiale laisse désormais la place à un nouvel oligopole, beaucoup plus concentré, de firmes mondiales [11]. Cette évolution s'accompagne de mutations structurelles caractérisées par des phénomènes d'intégration verticale et horizontale. Ainsi, l'érosion du rôle du négoce est compensée chez les plus grosses sociétés par leur diversification et leur entrée dans la première transformation. C'est le cas de Cargill et d'Archer Daniels Midland (ADM) qui, de leur métier de céréaliers, sont entrés (à des périodes différentes) dans le cacao puis le broyage, ou encore d'ED\&F Man. C'est aussi le cas de certains chocolatiers (comme Kraft Jacobs Suchard ou Nestlé) qui investissent dans les années 80 dans des activités de première transformation [12].

\section{Libéralisation des années 90 : recompositions ivoiriennes, enjeu et illustration des recompositions mondiales}

Au cours des années 90, l'économie ivoirienne connaît une libéralisation tardive mais à marche forcée. Un véritable programme de privatisations est mis en place dès 1991 pour désengager l'État des activités productives et de certains services. La dévaluation du franc CFA de 1994 conduit à une accélération du processus : en offrant une nouvelle rentabilité à de nombreuses activités et en permettant un dégrippage de l'économie, elle facilite l'investissement, en particulier étranger. En ce qui concerne la filière cacao et la caisse de stabilisation, qui représentent des enjeux stratégiques, les pouvoirs publics vont cependant pratiquer la résistance et organiser une lutte pied à pied avec les bailleurs de fonds internationaux. Elle durera dix ans, jusqu'à la suppression de la CSSPPA en janvier 1999.

Malgré les nombreuses péripéties liées à cette libéralisation, la Côte d'Ivoire va néanmoins poursuivre la croissance de sa production. Cette progression fait pourtant suite à l'onde de choc de la guerre du cacao et à une crise de confiance chez les producteurs ivoiriens. Elle révèle la puissance des mécanismes de la mise en valeur agricole sur front pionnier ${ }^{11}$ qui, à partir d'un libre accès à la terre et à la main-d'œuvre, permet le maintien de la dynamique de la "frontière ", malgré la baisse des revenus.

Après s'être stabilisée au-dessus du palier de 800000 tonnes à partir de la fin des années 80, la production, déjouant tous les pronostics, atteint le volume record de 1,25 million de tonnes en 19951996 dans un contexte de marché international qui est cette fois déficitaire ${ }^{12}$. La Côte d'Ivoire, qui avait depuis longtemps son statut privilégié de premier producteur mondial, devient dès lors - avec 45 $\%$ de l'offre - le lieu stratégique pour l'approvisionnement de l'industrie cacaoyère. Pour les grands groupes mondiaux, qui ont émergé dans les années 80 , il s'agit d'acquérir des positions de marché en Côte d'Ivoire pour sécuriser leurs approvisionnements, d'autant plus que le processus de libéralisation ouvre de nombreuses incertitudes. Cet objectif va rencontrer le besoin d'alliances des firmes ivoiriennes dont la physionomie est bouleversée par les conséquences de la libéralisation et les opportunités des privatisations. Et les stratégies des firmes nationales et étrangères vont aussi rencontrer la volonté du gouvernement d'augmenter la transformation locale. 


\section{Modalités du retrait de l'État}

Rappelons tout d'abord les conditions du désarmement du rôle de l'État dans la filière puisque, en changeant les règles du jeu, elles obligent les firmes à s'adapter. Le retrait de l'État a connu plusieurs étapes selon trois objectifs :

- la libéralisation de la commercialisation intérieure puis des exportations ;

- le principe « d'étanchéité » de chaque filière dans la gestion de la Caisse ${ }^{13}$;

- la diminution des coûts de mise en marché.

Pour atteindre ces objectifs, les mesures prises se sont inscrites dans trois directions.

II s'agit en premier lieu du démantèlement du système de prix et d'achat administré qui permet de libéraliser les prix intérieurs et les conditions de la collecte et de réduire les coûts d'une commercialisation désormais concurrentielle : après la baisse du prix garanti au producteur fin 1989 (la première depuis 25 ans), puis des quotas d'achat attribués aux exportateurs en 1990, les barèmes sont supprimés en 1991 et remplacés par la publication de trois prix (FOB, loco-magasin et bord champ). Le prix au producteur reste alors garanti puis devient indicatif (1995), pour être finalement supprimé en 1999.

La deuxième série de mesures concerne la réduction du rôle de la Caisse. Elle porte sur son retrait de la commercialisation intérieure (suppression des contrôles et de la gestion du coût du transport en 1991, puis de la gestion de la sacherie en $1996{ }^{14}$ ) et sur l'abandon progressif de ses prérogatives sur les exportations (mise en place d'un programme de ventes anticipées à la moyenne - PVAM - dès 1991, puis d'un système d'enchères géré par messagerie électronique en 1995 et, enfin, limitation des ventes directes de la Caisse à $15 \%$ du volume de la récolte en $1997^{15}$ ).

Il s'agit, enfin, de la professionnalisation de la gestion de la filière par la mise en place de plusieurs procédures de concertation et organes de pilotage avec le secteur privé (ouverture du conseil d'administration de la Caisse, délégations de service au groupement des exportateurs - le Gepex -, mise en place d'un comité interministériel de suivi du système de mise en marché en 1995 et nouvelle loi coopérative fin 1997, destinée à développer le rôle des organisations de producteurs).

Malgré ce désarmement progressif, l'État va bien sûr continuer à jouer de toute son influence jusqu'au bout (c'est-à-dire la libéralisation totale) en accordant à plusieurs reprises des passe-droits, au grand dam des bailleurs de fonds ${ }^{16}$, en favorisant l'installation de nouveaux exportateurs locaux sur des bases partisanes ou, encore, en pesant lourdement dans les recompositions et les alliances entre firmes.

\section{Besoins d'alliances des firmes}

Le démantèlement du système de commercialisation administré s'est traduit pour les firmes nationales et étrangères par une croissance de l'incertitude et du risque : incertitude des volumes et 
des coûts de collecte du cacao pour les exportateurs locaux, liée à la nouvelle situation de concurrence (fin des quotas d'achat et des prix garantis) ; incertitude également quant au financement des activités de commercialisation du fait du durcissement des conditions du crédit bancaire (conséquence de la fin des garanties qu'offrait le système administré aux banques locales) ; incertitude enfin des approvisionnements pour les négociants et les industriels étrangers, liée à la fin de la garantie de la Caistab sur les expéditions (puisque la Caisse couvrait les risques de défaillance des exportateurs).

La réponse des firmes a été la recherche d'alliances par des participations financières croisées ou des contrats d'approvisionnement. Ces alliances permettaient, dans le cas des entreprises ivoiriennes, d'accéder à des financements extérieurs en attestant de leur adossement à des grands groupes internationaux. Dans le cas des entreprises étrangères, elles permettaient d'effectuer une " remontée à l'origine ", c'est-à-dire d'accéder directement au cacao ivoirien, ou encore - et peut-être surtout d'éviter que les concurrents n'acquièrent des positions dominantes ou n'accèdent à des fèves moins chères. Il convient toutefois de mentionner un cas de figure particulier : celui de la firme française de transport Bolloré qui, sans être spécialisée dans le cacao, a aussi participé à ce jeu des alliances dans une logique non de contrôle du produit pour ses qualités intrinsèques mais de contrôle du fret ${ }^{17}$.

L'analyse des stratégies des firmes ivoiriennes doit préalablement prendre en compte l'importance des recompositions au sein du groupe des exportateurs au tournant des années 90 . En effet, la guerre du cacao et la crise économique du pays ont eu des conséquences sévères sur la santé financière de plusieurs sociétés qui ont été fragilisées. C'est le cas de JAG, deuxième exportateur ivoirien, qui doit cesser son activité en 1993 et est alors racheté par d'autres intérêts locaux. Parallèlement, certaines firmes, qui étaient encore sous contrôle extérieur, voient une montée en puissance des intérêts ivoiriens. C'est le cas de SIFCA, premier exportateur du pays qui passe sous contrôle exclusivement national. Ces évolutions et les opportunités des privatisations et de la dévaluation vont permettre l'émergence de groupes agro-industriels qui, à partir de leurs intérêts dans les filières cacao et café, vont investir progressivement d'autres activités agro-exportatrices (palmier, hévéa, coton), puis se diversifier dans d'autres secteurs jusqu'à former d'importants holdings parmi lesquels OCTIDE (issu de JAG) et SIFCOM (créé à partir de SIFCA) sont les plus connus ${ }^{18}$.

Ces nouveaux groupes diversifiés, tout comme les autres exportateurs, vont devoir chercher des financements externes non seulement du fait de la croissance des besoins financiers liée à l'augmentation de la récolte cacaoyère ivoirienne ${ }^{19}$, mais aussi du fait de la faiblesse des capacités de financement local. Les banques ivoiriennes sont en effet des banques sous contrôle étranger (filiales de banques françaises) qui ont toujours eu, traditionnellement, une activité de banque de dépôts plutôt que de banque d'affaires. Leur intervention dans le financement de la commercialisation s'effectuait grâce à des refinancements auprès de la banque centrale (la BCEAO) selon un système extrêmement sophistiqué couvrant l'essentiel du risque. Le retrait de la BCEAO du financement des campagnes, en conséquence de la fin des garanties qu'apportait le système administré, s'est traduit par un repli du financement local et par la recherche impérative de financements off shore qui étaient la condition sine qua non de maintien des positions commerciales.

Cette recherche d'alliances va se concrétiser par de nombreux montages techniques et financiers qui vont évoluer à un rythme soutenu tout au long de la décennie. L'articulation et l'intégration de la filière ivoirienne avec les firmes mondiales ont en effet soumis le cacao ivoirien aux conséquences des restructurations du négoce et de l'industrie au niveau international. Le secteur stratégique de l'économie est désormais pris dans le tourbillon de la mondialisation et de ses réactions en chaîne. 
Parmi les principales associations, il convient de citer :

- chez les industriels, l'entrée de Grace Cocoa dans le capital de SIFCA, en 1996 (les deux groupes étaient déjà associés dans le broyeur UNICAO) afin d'anticiper l'arrivée de Cargill ${ }^{20}$, le contrôle de l'exportateur Tropival par ED\&F Man, la participation de Cacao Barry au capital de la SHAC ;

- chez les négociants, l'implantation de plusieurs sociétés françaises (Orébi, Touton, GEPRO) qui s'associent ou contrôlent de petits exportateurs ivoiriens (ERAF, PRN, Delbau, SOGIMEX) ;

- dans le cas du transporteur Bolloré, le rachat de DAFCI puis de SHAC.

\section{Opportunité des investissements industriels}

Le développement accéléré de la capacité de broyage ivoirienne au cours des cinq dernières années apparaît comme atypique en regard de la structure traditionnelle du marché cacaoyer. En effet, les besoins des industriels de la chocolaterie portent sur des assemblages de beurres de différentes origines. Le broyage à l'origine est donc peu compétitif puisqu'il nécessite ensuite des mélanges complémentaires. Une possibilité serait éventuellement d'importer des fèves en provenance d'autres pays producteurs pour réaliser le mélange localement. Mais cette solution n'est pas réaliste du fait des surcoûts auxquels doivent déjà faire face les industries locales car les coûts industriels chez les pays producteurs sont généralement plus élevés : coûts des équipements et de l'assistance technique, des services et de l'énergie, qui dépassent les gains liés à la faible rémunération de la main-d'œuvre industrielle ${ }^{21}$. Les beurres fabriqués dans les pays producteurs de cacao sont également handicapés par leur qualité réputée inférieure à celle des beurres fabriqués dans les pays industriels. Cette réputation, qui n'est pas toujours justifiée, se traduit par une décote qui rend encore plus difficiles la rentabilité et la compétition sur le plan international ${ }^{22}$. La rentabilité du broyage local par rapport à une exportation en fèves de bonne qualité est ainsi souvent réduite. Dans tous les pays producteurs, elle a nécessité des aides publiques et des régimes fiscaux privilégiés destinés à compenser ces handicaps.

La géographie du broyage mondial au milieu des années 90 était la suivante : $50 \%$ en Europe (y compris l'ex-URSS), $13 \%$ aux États-Unis, $2 \%$ au Japon, $23 \%$ dans les pays producteurs et $11 \%$ dans les autres pays (Chine, Inde, Asie du Sud-Est). Parmi les pays producteurs, les 620000 tonnes produites provenaient du Brésil (29\%), de Côte d'Ivoire (23\%), de Malaisie (16\%), du Ghana (11\%), d'Indonésie (10\%), d'Équateur (6\%), du Nigeria (3\%) et du Cameroun (2\%). La chute de la production au Brésil et en Malaisie se traduit aujourd'hui par une surcapacité puisque le broyage équivaut à 90 et $70 \%$ respectivement de la production nationale et impose des importations de fèves. L'Équateur, qui avait mené une politique très volontariste de transformation locale dans les années 80 (jusqu'à $80 \%$ de la production transformée), a été obligé de fermer de nombreuses usines. Quant aux pays africains, ils avaient conservé au cours des dernières décennies une capacité de broyage équivalant à environ $20 \%$ de leur production nationale.

L'évolution de la situation ivoirienne doit s'analyser en regard de cette configuration générale. Nous avons vu que les choix originels découlaient d'une volonté de développement industriel national et, 
surtout, de valorisation de la filière. La forte progression de la production à partir des années 80 s'est traduite par une sous-capacité de transformation des fèves de mauvaise qualité (principalement les petites fèves de la récolte dite intermédiaire qui fait suite à la récolte principale d'octobre à janvier). Avec 113000 tonnes, la capacité de broyage ivoirienne était tombée à $10 \%$ de la production nationale : les investissements des années 1995-2000 sont donc d'abord des investissements de rattrapage destinés à remettre à niveau l'outil industriel pour une meilleure valorisation de la filière. Ainsi, SACO a porté ses capacités de 70000 à 100000 tonnes, UNICAO de 43000 à 86000 tonnes et deux nouveaux opérateurs se sont installés : le chocolatier français Cantalou avec 60000 tonnes et, enfin, l'américain Cargill avec une unité de 60000 tonnes qui doit être portée contractuellement à 100000 tonnes. Soit une progression de 233000 tonnes qui représente dans un avenir proche (dès l'extension de l'usine Cargill) un ratio de broyage local de l'ordre de $30 \%$.

Si le rattrapage de l'outil industriel s'explique par ces agrégats globaux, il a cependant évidemment été l'occasion de stratégies croisées du gouvernement ivoirien, des firmes locales et des firmes étrangères. Pour l'État, dont l'ambition affichée depuis 1996 est d'atteindre 500000 tonnes de broyages (ce qui semble irréaliste), cet enjeu industriel a fait l'objet d'un soutien actif pour deux raisons principales et liées.

* Il s'agit tout d'abord d'essayer de sortir de la trop grande dépendance du marché international de la fève. L'échec cuisant de la stratégie de rétention de la guerre du cacao a montré l'impossibilité d'une action directe sur les cours. Le recours au broyage offre ainsi deux avantages : une volatilité légèrement moins forte liée à l'effet de lissage (délais de transformation et stockage plus facile) et, surtout, une meilleure intégration dans le jeu des industriels mondiaux avec l'accès à un pouvoir de négociation plus significatif, même si celui-ci reste limité. La volonté d'augmentation de la capacité de broyage a ainsi été utilisée comme un droit d'accès ou un ticket d'entrée sur le marché ivoirien des firmes étrangères qui, on l'a vu, souhaitaient se positionner chez le premier producteur mondial.

* La seconde raison était ainsi de forcer les grands opérateurs mondiaux et les industriels étrangers à investir en Côte d'Ivoire pour modifier l'équation de l'insertion ivoirienne dans le marché. L'opposition du gouvernement à accepter l'installation de Cargill pendant près dix ans, jusqu'à ce que celui-ci verse enfin le gage d'un investissement industriel, en est une bonne illustration.

Pour les firmes, les raisons du développement industriel facilité par les avantages fiscaux (même si ceux-ci ont progressivement été remis en cause) ${ }^{23}$ relèvent de deux objectifs principaux. Le développement du broyage a bien sûr été l'occasion de consolider les stratégies d'alliances. La participation de Grace Cocoa à la création de la seconde tranche d'UNICAO en 1995 facilite ainsi sa contribution à l'augmentation de capital de SIFCA en 1996. Mais elle permet aussi aux firmes étrangères de renforcer leurs positions pour l'accès aux fèves ivoiriennes. C'est le choix de Cacao Barry avec SACO - qui est désormais contrôlé à $100 \%$, suite au désengagement de l'État - ou encore de Cargill, qui augmentent ainsi leurs capacités, directement sur place, chez le premier producteur mondial. C'est aussi celui du chocolatier Cantalou. Les industriels du chocolat n'ont cependant pas tous cette stratégie puisque Nestlé, l'un des plus grands chocolatiers - installé sur place avec son usine de café soluble depuis 1959 -, n'a pas participé à cette course aux capacités de broyage locales. Parallèlement, les exportateurs ivoiriens ont poursuivi leur logique d'intégration en renforçant leurs positions industrielles. C'est l'option suivie par SIFCA, et OCTIDE essaiera sans succès (cf. infra) de racheter Cacao Barry lors de sa mise en vente en 1996.

La stratégie mise en œuvre par SIFCA mérite d'être détaillée car son caractère extrêmement 
volontariste revêt un caractère exceptionnel dans l'environnement économique africain. II est aussi exemplaire des logiques à l'œuvre dans le monde de l'industrie. En effet, le groupe a fait le choix de poursuivre son intégration verticale à partir de son usine UNICAO en s'adaptant le plus possible aux nouvelles caractéristiques de la demande des chocolatiers. À partir de sa production de beurre, il s'est lancé dans la production de beurre de cacao liquide qui correspond aux nouveaux standards d'approvisionnement de la chocolaterie, tout en offrant une valeur ajoutée supplémentaire. La grande originalité pour une entreprise ivoirienne - et une première pour un pays producteur - vient du lieu de réalisation de l'investissement industriel, puisque c'est un site européen qui a été retenu afin d'être plus proche des utilisateurs. La société Nord Cacao a ainsi été créée près de Dunkerque dans le Nord de la France en 1993 par SIFCOM (le holding de contrôle de SIFCA) en association avec une société d'ingénierie spécialisée britannique. Avec une capacité de traitement annuelle de 18000 tonnes portée à 40000 tonnes en 1998, Nord Cacao produit du beurre liquide, filtré, désodorisé et livré en camions-citernes, en flux tendu, auprès de plusieurs chocolatiers européens dont Jacobs Suchard, ce qui constitue une référence de qualité. Dans la mesure où les chocolatiers utilisent des mélanges d'origines, la production de Nord Cacao repose à $50 \%$ sur du beurre UNICAO et à $50 \%$ sur d'autres provenances en fonction des demandes des clients.

La logique industrielle du groupe SIFCOM a été poursuivie en intégrant également la poudre de cacao à sa chaîne de production. Pour des raisons techniques, UNICAO avait initialement choisi de ne pas fabriquer de poudre et d'exporter directement les tourteaux ${ }^{24}$. Après avoir envisagé une unité de production sur le site de Nord Cacao, le groupe a finalement retenu une alliance industrielle puis la prise de contrôle du fabriquant de poudre espagnol INDCRESA qui est approvisionné par UNICAO et opère des mélanges avec des tourteaux d'origine moins onéreuse (comme la Malaisie). Enfin, le doublement de la capacité d'UNICAO en 1995 s'est accompagné d'un choix industriel haut de gamme avec recours aux technologies les plus modernes, à l'occasion duquel l'ensemble de l'entreprise a été mise aux standards exigés par les utilisateurs européens. Le process de la nouvelle unité est entièrement informatisé et permet de proposer des produits spécifiés selon les critères requis par les clients. Alors que la première usine UNICAO fabrique à $90 \%$ du beurre et des tourteaux, la nouvelle unité fabrique de la masse, ce qui est rare chez les pays producteurs ${ }^{25}$.

Cette relecture des recompositions industrielles de la filière ivoirienne doit enfin être complétée par celle des recompositions du broyage mondial qui affectent directement la physionomie des rapports de force en Côte d'Ivoire. La fin de la décennie 90 voit en effet l'accélération du phénomène de concentration engagé dans les années 80 .

La première opération d'envergure a été la vente, en 1996, de l'industriel d'origine française Cacao Barry, propriétaire de SACO, par son holding de contrôle ${ }^{26}$. L'annonce de la vente déchaîne évidemment les appétits étrangers, mais le dossier prend également une tournure très politique en Côte d'Ivoire du fait des implications sur le contrôle de SACO. Le groupe OCTIDE, qui détient JAG, se met aussitôt sur les rangs afin d'engager son intégration verticale, à l'instar de ce qui a été réalisé par SIFCA avec UNICAO dix ans plus tôt. La présidence ivoirienne apporte son soutien et espère mobiliser aussi les autorités françaises, en répétant le scénario de la reprise de JAG. Mais le coût de la transaction écarte les ivoiriens et Barry est finalement acquis pour 200 milliards de francs CFA (350 millions d'euros) par le holding suisse Klaus Jacobs via sa filiale allemande Callebaut. Le nouveau groupe Barry-Callebaut, qui mise sur la complémentarité broyage-chocolat de couverture (spécialité de Callebaut), devient le premier industriel mondial du cacao. Le second épisode de l'année 1996 est la vente par le groupe chimique américain Grace de sa branche cacao, motivée par une volonté de 
recentrage sur ses métiers de base. Sa filiale Grace Cocoa contrôle plusieurs broyeurs (dont le hollandais De Zaan et l'américain Ambrosia). Après plusieurs hypothèses de rachat partiel par Nestlé et Cargill, la surprise est venue du céréalier ADM, qui acquiert Grace Cocoa pour 270 milliards de FCFA et réalise ainsi son entrée royale dans le cacao. ADM trouve dans la " corbeille de la mariée " non seulement la participation de De Zaan dans UNICAO mais aussi les $30 \%$ que Grace avait acquis six mois plus tôt dans SIFCA, premier exportateur ivoirien. ADM consolide sa position quelques mois plus tard en rachetant la division industrielle du négociant britannique ED\&F Man et ravit la place de leader mondial de la transformation à Barry-Callebaut. En Côte d'Ivoire, SIFCA est son associé.

Au début du nouveau siècle, le palmarès du broyage mondial serait désormais : ADM avec 530000 tonnes, Barry Callebaut avec 385000 tonnes, Cargill (345 000 tonnes) et les américains Hamester (165 000 tonnes) et Blommer (110 000 tonnes). Pour les chocolatiers les premiers rangs sont les suivants : Nestlé (Suisse) 230000 tonnes, Hershey (États-Unis) 90000 tonnes, Cadbury (RoyaumeUni) 85000 tonnes, Mars et Philip Morris ${ }^{27}$ (États-Unis) 80000 tonnes. Côte d'Ivoire exceptée, les premiers groupes pèsent désormais plus que les principaux pays producteurs de cacao.

\section{Modalités et incertitudes des formes de coordination après la libéralisation}

La libéralisation complète de la filière cacao ivoirienne et la suppression de la caisse de stabilisation en 1999 ont bien sûr entraîné de nombreuses et nouvelles conséquences sur la physionomie du secteur. Les stratégies mises en œuvre par les firmes nationales et étrangères au cours des années 90 dans un contexte de profonde restructuration du marché mondial ont été exacerbées au cours des deux dernières années. Elles se sont traduites par de nouvelles recompositions qui ont vu l'éviction ou la prise de contrôle des principales entreprises ivoiriennes par les grandes firmes mondiales.

\section{Tentatives de rénovation de la commercialisation intérieure}

Le passage du système de traite d'un régime administré, avec des quotas d'achat et des prix fixés par un barème à chaque étape de la commercialisation, à un système de marché libre s'est traduit par une croissance du risque lié aux incertitudes sur les prix et l'approvisionnement.

La réduction des coûts de transaction inhérents à l'incertitude du marché spot peut être réalisée selon deux voies : soit l'intégration verticale, où la firme internalise complètement la prise de risque, soit la mise en œuvre de contrats entre les différentes catégories d'agents de la chaîne de production. Dans le cas de l'activité agricole, il existe plusieurs types de formes contractuelles correspondant à des degrés de sophistication croissants. Selon Mighell et Jones [13] ou encore Jaffee [14], on peut ainsi distinguer principalement : la vente fidélisée (garantissant l'écoulement), la vente à terme (garantissant l'écoulement à un prix, une date et une quantité définis à l'avance), le contrat avec spécification de marché (qui délègue à l'acheteur les décisions et le risque commercial sur les dates et lieux de vente), le contrat de gestion (où certaines décisions relatives à la gestion technique de la production sont aussi déléguées à l'acheteur) et, enfin, le contrat avec fourniture de ressources (où l'acheteur fournit également des inputs nécessaires à la production).

Au cours de la dernière décennie, plusieurs exportateurs ivoiriens se sont efforcés d'innover par rapport au système classique de la traite fondé sur la confiance et les avances de trésorerie en améliorant leurs réseaux d'achat. Leur objectif était de sécuriser leurs approvisionnements dans un contexte où il existait encore un prix indicatif au planteur. Mais la fin des prix intermédiaires (liée à la suppression du barème) et le tarissement des sources traditionnelles de financement bancaire fragilisaient le dispositif d'avances de trésorerie et incitaient à une meilleure performance 
commerciale par la réduction des coûts. Les incertitudes liées aux bouleversements du monde de l'export renforçaient aussi l'intérêt d'une consolidation du système d'approvisionnement.

Trois tentatives correspondant à un degré de formalisation croissant ont été réalisées.

* La première a pris la forme d'une centrale d'achat fondée en 1994 par sept petits exportateurs (société $\mathrm{ACCACl}$ ). Cette centrale assurait le groupage des produits et leur conditionnement pour le compte de ses exportateurs associés. Tout en restant très souple, elle offrait un niveau intermédiaire entre le monde de la traite et les exportateurs : par le regroupement des moyens ${ }^{28}$, elle évitait la juxtaposition de dispositifs concurrents et la " course au produit ». ACCACl a ainsi développé un réseau d'approvisionnement fidélisé en proposant des contrats de livraison et un appui technique. Elle travaillait aussi bien avec les traitants traditionnels qu'avec les organisations coopératives et drainait pour le compte de ses membres de l'ordre de 100000 à 120000 tonnes.

* La deuxième expérience a été développée à l'initiative de SIFCA à partir de 1990. Elle correspond à une construction beaucoup plus formalisée, eu égard à la rusticité des relations existantes dans le cadre de la traite traditionnelle, associant groupements de producteurs et exportateurs. Elle reposait sur un montage juridique sophistiqué avec la création de sociétés anonymes à capital variable, appelées SOGEPAG, regroupant plusieurs organisations coopératives dont les parts sociales évoluaient en fonction des tonnages livrés. Le système reposait en outre sur la mise à disposition d'un encadrement technique et d'équipements (véhicules de transport, magasins, matériel de pesée, mais aussi décortiqueurs pour le café) et d'un dispositif de paiement au comptant permettant un écoulement garanti au prix officiel, majoré de ristournes proportionnelles aux résultats. Le dispositif a connu un bon développement entre 1990 et 1995. Dix-sept SOGEPAG ont été mises en place et le réseau a réussi à atteindre 50000 tonnes de cacao (et 10000 tonnes de café).

* La troisième expérience, enfin, est la plus atypique mais aussi la plus marginale. Elle correspond cette fois à un processus d'intégration verticale de la production par un négociant. Elle a été engagée par la société française Touton en 1996 et marque une tentative tout à fait nouvelle dans le contexte ivoirien de création d'une chaîne intégrée de production de cacao de qualité. Elle comprend deux volets : d'une part, un volet "production directe ", avec la création de plantations de cacaoyers hybrides sous ombrage, et, d'autre part, un volet " préparation du produit », avec l'installation de deux centres de traitement post-récolte assurant la fermentation puis le séchage artificiel des fèves. Ce dispositif intégré repose sur deux opérateurs :

- la Société agricole de l'Ouest (SAO), créée à l'initiative de Touton, qui possède et gère les plantations et les centres de traitement post-récolte ${ }^{29}$;

- l'exportateur Delbau, contrôlé aujourd'hui par Touton, qui assure l'expédition directe du produit en France.

Il permet, grâce à un cahier des charges et au contrôle de qualité assuré par la société de négoce, de préparer des lots de cacao homogènes et spécifiés et d'assurer une parfaite traçabilité du produit. Cette approche unique en Côte d'Ivoire esquisse une éventuelle segmentation du système d'offre en réponse à la segmentation, qui reste marginale, des marchés de consommation. 
À l'exception de cette dernière formule qui est extrêmement limitée, les autres expériences sont restées des tentatives sans suite. Les déboires des exportateurs nationaux et, surtout, les coûts de fonctionnement d'un dispositif comme les SOGEPAG n'ont pas permis de résister à la concurrence par les prix induite par la mise en œuvre de la libéralisation. Dans un contexte où les producteurs ont connu une érosion considérable de leurs revenus depuis le milieu des années 80 , le prix d'achat reste le critère déterminant et les planteurs sont beaucoup plus sensibles aux surenchères des traitants qu'à la mise à disposition d'infrastructures et d'encadrement technique. Ce type de dispositif n'est possible qu'en situation de marché déprimé, lorsque l'écoulement des produits par les planteurs est difficile ; il ne peut en aucun cas résister à la flambée de la concurrence.

Ainsi, aujourd'hui, la commercialisation ivoirienne reste très fruste et soumise à la loi du collecteur de brousse qui pousse ses achats et propose ses prix en fonction des directives de son exportateur donneur d'ordre. Les firmes exercent leur concurrence à travers la fidélisation de leurs réseaux de traitants et non par celle des producteurs, par leur capacité à mettre en place la trésorerie nécessaire aux achats et par leur capacité et leur rapidité à " coller » aux prix de marché. La course au produit est la règle, les producteurs pouvant y gagner ponctuellement, et entraîne parfois des baisses significatives de la qualité. Celle-ci, malgré une plus grande instabilité, reste globalement stable avec cependant une baisse tendancielle au cours des dernières années ${ }^{30}$.

Ainsi, les campagnes ivoiriennes, après la parenthèse du système administré sous l'égide de la Caisse qui a duré 45 ans, sont retournées au fonctionnement originel de la traite coloniale. Seules l'époque et les caractéristiques des exportateurs ont changé. Les perspectives ouvertes par la nouvelle loi coopérative ne pourront avoir d'effet qu'à moyen terme et les organisations de producteurs ont un lourd handicap à surmonter, hérité de plusieurs décennies de mise sous tutelle de l'État. Elles sont très loin de pouvoir intervenir dans le jeu de leurs compétiteurs directs que sont les grandes firmes mondiales du cacao.

\section{Nouvelles modalités d'intégration au marché mondial}

Les principales incertitudes de la filière ivoirienne proviennent aujourd'hui des conditions de son intégration au marché mondial et des conséquences de la libéralisation qui s'est traduite par deux phénomènes :

- une baisse des prix mondiaux, qui s'est répercutée au niveau du producteur, contredisant ainsi les objectifs des tenants de la libéralisation ${ }^{31}$;

- une forte concentration des exportateurs avec une marginalisation radicale des firmes nationales.

La relation entre baisse des cours et libéralisation en Côte d'Ivoire est bien sûr controversée. Deux écoles s'affrontent pour interpréter cette chute ${ }^{32}$ : celle des « fondamentaux » du marché avec une surproduction au niveau mondial dont la Côte d'Ivoire serait largement responsable ; celle de l'impact de la libéralisation ivoirienne sur le marché, la suppression de la Caisse et de la stabilisation ayant entraîné la fin des ventes anticipées et une concentration des transactions en spot entre novembre et février, avec un effet dépressif sur les cours. Comme bien souvent, la vérité est dans un entre-deux. Cependant, si les dernières campagnes ont effectivement vu une poussée de l'offre de la Côte d'Ivoire, mais aussi de l'Indonésie et du Ghana (atténuée par la chute du Brésil), le vrai changement structurel reste bien la libéralisation chez le premier producteur mondial. Le marché cacaoyer est ainsi passé d'une demi-libéralisation à une libéralisation quasi complète, puisque seul le Ghana (10\% du marché) continue la résistance avec son Cocoa marketing board contrôlé par l'État. 
Le panorama de l'exportation a, quant à lui, été bouleversé par l'implosion du groupe SIFCOM qui était le principal holding ivoirien et dont on a vu les remarquables efforts d'intégration industrielle. En effet, la croissance extrêmement forte du groupe depuis le milieu des années 90 s'était traduite par une augmentation rapide de sa fragilité financière. Son rachat de JAG à OCTIDE en 1999 en avait fait un Goliath du cacao aux besoins en trésorerie considérables, nécessaires à l'achat d'environ 350000 tonnes de fèves. Ceux-ci n'étaient satisfaits qu'au coup par coup, et avec beaucoup de réserves, par les banques internationales et par son nouvel actionnaire ADM, grâce à sa forte assise politique et sa proximité de la présidence de la République. Le coup d'État de décembre 1999, la nouvelle donne politique ivoirienne et surtout de graves erreurs commerciales dans la stratégie de vente au printemps 2000 (portant sur 250000 tonnes) ont précipité la chute. SIFCOM a dû revendre ses différentes filiales par appartements.

SIFCA, UNICAO, Nord Cacao et les autres sociétés de son empire cacaoyer ont bien sûr été reprises par ADM qui a ainsi consolidé son statut de firme leader du marché. Ce coup de tonnerre s'est accompagné au cours de l'année 2000 par une marginalisation de l'ensemble des petits exportateurs locaux qui n'étaient plus en mesure d'accéder aux financements, faute de garanties du système de stabilisation. Et certaines grandes firmes mondiales auraient essayé de faire place nette fin 2000 en se lançant dans une surenchère sur les achats pour mettre à terre les derniers exportateurs nationaux $[16]^{33}$. Lors de la dernière campagne cacaoyère, moins d'une dizaine d'exportateurs étrangers, ou sous contrôle étranger, parmi lesquels ADM, Cargill, Barry-Callebaut, DAFCl-Bolloré, Tropival-Newco, Delbau-Touton ${ }^{34}$, auraient ainsi réalisé $85 \%$ des expéditions.

Une telle évolution s'est bien sûr traduite par une réaction du nouveau gouvernement ivoirien issu de la transition de l'année 2000 et, sous la pression des planteurs qui représentent environ 4 millions de personnes et des responsables des sociétés locales, l'État s'est engagé dans un processus visant à la remise en place d'un prix minimum au producteur et à une réglementation de la concurrence destinée à limiter les positions de marché. Une étude a été confiée à la banque d'affaires britannique HSBC qui propose plusieurs options dont la création d'un système de stabilisation privé, géré par la profession, sur la base du système des ventes anticipées à la moyenne et d'une couverture du risque sur le marché à terme. Le gouvernement a créé en juillet une Bourse du café et du cacao (BCC) qui sera chargée de réguler les exportations et leur répartition entre les différentes firmes. La complexité du système ainsi que le rôle dévolu aux représentants des producteurs, dont les compétences techniques sont très limitées, laissent aujourd'hui planer la plus grande incertitude sur la gestion future de l'offre ivoirienne.

Notes :

${ }^{1}$ Adaptation française d'un article à paraître dans un numéro spécial du Journal of Agrarian Change : « Global commodity chains and African export agriculture » (vol. 2, n², 2002).

${ }^{2}$ Après torréfaction et broyage, les fèves sont transformées en masse (ou pâte ou liqueur) qui, après pressage, permet d'obtenir pour moitié du beurre et de la matière sèche (tourteaux). Les tourteaux sont ensuite transformables en poudre de cacao. Les ratios techniques sont : pâte/fèves $80 \%$, beurre/fèves $40 \%$, tourteaux/fèves $40 \%$.

${ }^{3}$ UNICAO est une joint venture associant l'exportateur SIFCA, le négociant français Tardivat et l'industriel néerlandais De Zaan (du groupe américain Grace Cocoa).

${ }^{4}$ Fabriquée par l'usine de café soluble CAPRAL qui produit du Nescafé depuis 1959. 
${ }^{5}$ Sur ce thème, voir Losch [3] qui développe la formation historique du complexe café-cacao ivoirien. Dans cette recherche, l'analyse du complexe politico-économique a été étendue au secteur café puisque sa croissance a participé du même phénomène, avec les mêmes institutions et les mêmes acteurs. L'ensemble des données factuelles sur l'évolution de la filière cacao présentées dans cet article provient de cet ouvrage. Elles ont été complétées pour les deux dernières années par l'analyse de la presse spécialisée et par des entretiens avec des professionnels de la filière.

${ }^{6}$ C'est notamment le cas des nouveaux producteurs d'Asie du Sud-Est - Malaisie et Indonésie - qui refuseront de participer à l'Accord. Pour une analyse des difficultés des accords internationaux, voir l'exemple de l'Accord international sur le café étudié par Bates [5].

${ }^{7}$ Les États-Unis, principal pays consommateur, font de même, vidant ainsi l'Accord de sa substance. La Côte d'Ivoire reviendra dans l'Accord en 1986, sous la pression de la France, mais le marché sera alors en pleine tourmente.

${ }^{8}$ (400 000 tonnes) avec la société française Sucres et Denrées et 280000 tonnes avec la société américaine Phillip Brothers (Phibro).

${ }^{9}$ Le Brésil a utilisé pendant plusieurs décennies une stratégie d'offreur résiduel sur le marché du café, qui a largement contribué au bon fonctionnement de l'Accord international sur le café, grâce à sa part de marché longtemps supérieure à $50 \%$ mais aussi parce que sa structure économique plus diversifiée lui permettait d'en supporter le prix [5, 7].

${ }^{10}$ Au moment de la guerre du cacao, la Côte d'Ivoire produit 800000 tonnes mais le montant des contrats au niveau mondial, physique et terme confondus, représente de l'ordre de 20 millions de tonnes, soit près de huit fois la production mondiale.

${ }^{11}$ Les systèmes sur front pionnier sont caractérisés par une extension continue de la frontière agricole par défrichement du couvert forestier. Ils ont été une figure majeure de la croissance de la production dans de nombreux pays tropicaux.

${ }^{12}$ Entre 1988 et 1996, la production mondiale n'a progressé que de 1,5\% par an alors que celle des broyages a été de 3,5\%. Cette évolution, qui se traduit par une baisse des stocks mondiaux et une pression sur les prix, s'explique pour partie par l'effondrement des productions brésilienne et malaisienne.

${ }^{13}$ La CSSPPA a assuré le contrôle de plusieurs filières d'exportation (jusqu'à neuf à la fin des années 70). La pratique était de consolider les résultats et de subventionner les filières déficitaires par les filières bénéficiaires selon la conjoncture des marchés. Au début des années 90 , ce principe était toujours en vigueur pour le café et le cacao.

${ }^{14}$ La Caisse assurait la mise à disposition des sacs nécessaires à la collecte puis au transport intérieur du cacao, soit environ 30 millions de sacs de jute de 60 kilos !

${ }^{15}$ Selon le principe des caisses de stabilisation, les exportateurs privés restaient propriétaires du cacao mais devaient obtenir l'autorisation de la Caisse pour exporter. À compter de la fin des années 70 , la Caistab avait cependant développé des ventes dites directes où elle négociait elle-même les contrats et demandait leur exécution aux exportateurs privés. Cette pratique avait atteint des niveaux très importants à la fin des années 80 (jusqu'à $80 \%$ de la récolte). 
${ }^{16}$ Le plus bel exemple, et le plus brutal, est l'organisation en 1999 - soit neuf ans après la fin des quotas - d'exportations hors du système d'enchères, avec attribution de tonnages d'expédition aux entreprises les plus proches du pouvoir.

${ }^{17}$ Dans ce cas, la remontée à l'origine vise à rentabiliser au maximum les investissements dans le secteur de la logistique et du transport. Dans plusieurs pays africains, Bolloré contrôle ainsi des lignes maritimes, le transit portuaire, le conditionnement des produits, les chemins de fer et, enfin, des plantations qui permettent de garantir du fret et de rentabiliser ses équipements.

${ }^{18}$ SIFCOM était devenu, fin 1999 et avant ses déboires récents, le premier groupe à capitaux africains (hors Afrique du Sud) dans les matières premières agricoles. Son chiffre d'affaires consolidé, en intégrant ses activités dans l'importation, la distribution, la pharmacie, les télécommunications et les services, avoisinait les 1000 milliards de francs CFA (environ 1,5 milliard d'euros).

${ }^{19}$ Le saut de la récolte de 400000 tonnes puis la hausse des cours des années 1994-1997 entraînent un coût annuel de mise à FOB de la récolte de près de 900 milliards de francs CFA (1,3 milliard d'euros). La filière cacao est le premier secteur d'activité des banques ivoiriennes et représente jusqu'à $25 \%$ de leur encours total.

${ }^{20}$ L'implantation de Cargill en Côte d'Ivoire a été le " serpent de mer " de la filière pendant presque une décennie. En effet, la société américaine a eu beaucoup de difficultés à prendre pied sur le territoire ivoirien car elle était perçue par les pouvoirs publics comme un danger potentiel. Houphouët-Boigny interviendra personnellement, appuyé par le gouvernement français, pour éviter le rachat de JAG par Cargill en 1993.

${ }^{21}$ Les surcoûts liés aux nouvelles réglementations sur l'environnement dans les pays industrialisés pourraient éventuellement offrir un nouvel avantage aux pays producteurs qui ne sont pas soumis aux mêmes contraintes.

${ }^{22}$ II n'existe pas de cotation pour le beurre de cacao. Le ratio de prix habituellement retenu entre beurre et fève est de 2,7. Les décotes selon les origines et la réputation des industriels peuvent être très élevées et l'écart peut aller jusqu'à près de $100 \%$ entre, par exemple, un beurre fabriqué aux États-Unis et un beurre nigérian.

${ }^{23}$ Sous la pression des bailleurs de fonds, les subventions directes étatiques ont été supprimées en 1998. Une taxe d'exportation préférentielle a cependant été maintenue pour les produits des broyages.

${ }^{24}$ La fabrication de poudre à l'origine dans les pays producteurs est handicapée par le taux d'hygrométrie qui entraîne un phénomène de fonte et des décotes de prix.

${ }^{25}$ Les chocolatiers sont réticents à acheter de la masse dans les pays producteurs car, contrairement au beurre, elle ne peut pas être désodorisée. La qualité nécessite une bonne maîtrise technique, notamment de la torréfaction.

${ }^{26}$ Repris initialement (1980) par le négociant français Sucres \& Denrées puis revendu par celui-ci en 1991 après ses déboires de la guerre du cacao, Barry appartient alors pour $51 \%$ à la SCl et pour $49 \%$ au groupe belge Albert Frère (Bruxelles-Lambert). La Société centrale d'investissements ( $\mathrm{SCl}$ ) est une société holding contrôlée à parité par la famille Vernes (de la banque française du même nom) et par le groupe italien Montedison. 
${ }^{27}$ Philip Morris est le holding de contrôle de KJS (Kraft - General Food - Jacobs Suchard). Jusqu'en 1989, Jacobs Suchard appartenait au groupe suisse Klaus Jacobs qui a choisi de se recentrer sur la première transformation et le chocolat de couverture avec sa filiale Callebaut. Ce recentrage explique la reprise du broyeur français Cacao Barry en 1996.

${ }^{28}$ Chaque exportateur annonçait en début de campagne ses objectifs de commercialisation et affectait à la centrale d'achat les préfinancements nécessaires à la collecte.

${ }^{29}$ La SAO possède 4000 ha. En 1999, 900 ha étaient déjà plantés, dont 250 ha en production. Cette technique de plantations en blocs, hors agriculture familiale, est rarissime en Côte d'Ivoire. Dans l'attente de la montée en production de ses plantations, la SAO achète aux planteurs de la région. Les fèves hors normes font l'objet d'un traitement post-récolte spécifique.

${ }^{30}$ La proportion de fèves de grade 1 (qualité supérieure) est passée de 70 à $50 \%$ entre 1997 et 2000 [15].

${ }^{31}$ La répercussion de cette baisse a été sévère puisque le prix au producteur est passé du niveau indicatif de $570 \mathrm{FCFA} / \mathrm{kg}$ lors de la campagne agricole 1998-1999, la dernière de la stabilisation, à 220$260 \mathrm{FCFA} / \mathrm{kg}$ début 2000 pour remonter à 400 FCFA en fin d'année. Au cours de la campagne 20002001, les prix ont connu une forte instabilité ainsi qu'une variabilité inter-régionale marquée [15].

${ }^{32}$ Le marché du cacao s'est « retourné » dès le début 1999 et l'an I de la libéralisation en Côte d'Ivoire a été salué par un effondrement des cours (de 901 f/tonne en février 1999 à 545 f en décembre).

${ }^{33}$ Selon certaines sources, certaines majors auraient aussi essayé d'étouffer les petits exportateurs locaux ne disposant pas d'infrastructures de conditionnement, en les mettant en défaut sur leurs expéditions en bloquant toutes les capacités industrielles de préparation des fèves à l'export pendant deux mois et demi. Les capacités de traitement de Bolloré, qui contrôle via sa filiale SAGA 60 \% du conditionnement local (mais est aussi exportateur), auraient été réservées par contrat et les grandes firmes mondiales auraient ensuite payé les frais de dédit pour non-exécution.

${ }^{34}$ Newco est l'ex-branche cacao d'ED\&F Man.

\section{CONCLUSION}

Le marché du cacao achève aujourd'hui une recomposition engagée dans les années 80 . Au terme de cette relecture de la place et du rôle de la filière cacaoyère ivoirienne, nous pouvons proposer plusieurs commentaires complémentaires et généraux.

Tout d'abord l'oligopole d'États-nations, qui avait été déstabilisé puis démantelé par le mouvement de libéralisation de l'économie mondiale, a perdu l'un de ses derniers prestigieux vestiges. L'oligopole de firmes qui lui a fait place est confirmé et marqué par un degré de concentration très important (de l'ordre de $60 \%$ ). Non seulement les premiers groupes mondiaux pèsent plus que les principaux pays producteurs de cacao, Côte d'Ivoire exceptée, mais ils contrôlent aussi aujourd'hui les fèves et les broyages ivoiriens.

Ensuite, la séparation des métiers entre industries de la chocolaterie et du broyage a été acquise au cours de la dernière décennie, alors que les années 80 étaient encore incertaines. Les premières se concentrent sur le marketing du produit final (conditionnement, publicité) alors que les secondes travaillent de plus en plus sous contrat pour la fourniture de matières premières à bas prix. Ainsi, les 
grands chocolatiers (Nestlé, Philip Morris, Mars) se sont séparés de l'essentiel de leurs industries de première transformation et ce sont les principaux broyeurs (ADM, Cargill, Barry-Callebaut) qui sont montés à l'assaut du contrôle de la production à l'origine. Cette remontée à l'origine se limite cependant aux ports et aux industries locales et s'appuie sur des réseaux de collecte en sous-traitance.

La priorité pour l'obtention de beurre à bas prix tire le marché vers des standards de qualité moyens à faibles qui sont compensés par des mélanges ad hoc et une plus grande performance technique des process industriels. L'évolution du marché vers une segmentation plus prononcée, à l'instar du marché du café, reste très limitée et des initiatives comme celles de la société Touton en Côte d'Ivoire devraient rester très marginales. La faible qualité du cacao standard, en limitant l'incertitude sur la qualité intrinsèque du produit, ne favorisera pas des modifications majeures des systèmes techniques de production ni des formes de coordination dans les filières. Le principal facteur d'évolution pourrait provenir de l'accroissement des normes sanitaires qui entraînerait alors la mise en place de dispositifs de production et de commercialisation adaptés.

L'existence d'un oligopole de firmes, la fin des systèmes centralisés d'offre ainsi que l'orientation de la demande vers un " cacao tout-venant mondial " marquent une érosion très forte, voire la fin du national et de la notion de "pays producteur ", sur le marché du cacao. Les anciens standards nationaux sont désormais remplacés par la réputation des broyeurs à respecter les commandes des firmes chocolatières (en temps, volume et qualité). Mais le processus d'intégration des firmes de broyage vers l'amont, par le contrôle des sociétés d'exportation locales, se traduit aussi par une forme d'érosion de la notion même de marché international, puisque les transactions sont désormais largement internalisées au sein des grands groupes mondiaux. Leur degré de concentration exclut tout arrangement au niveau mondial, mais il rend a priori possibles des comportements de collusion (tacite ou formelle) dans chaque marché national de consommation, à l'instar de l'évolution récente aux États-Unis. Désormais, les firmes globales sont les acteurs dominants et il est remarquable de constater qu'elles agissent aussi pour essayer d'influencer la géographie de la production. Cette perspective semble déjà bien engagée puisque, au cours de la dernière décennie, inquiètes de la dépendance du marché du cacao vis-à-vis de la Côte d'Ivoire, plusieurs grandes firmes ou leurs associations se sont impliquées dans des programmes de développement ou de relance de la production : Barry-Callebaut au Mexique, ED\&F Man au Nigeria, I'Association américaine des producteurs de chocolat au Pérou, au Panama et au Viêt-nam.

Enfin, pour les pays producteurs de matières premières qui sont restés par trop dépendants de leurs exportations, ces profondes recompositions conduisent, bien sûr, à des situations particulièrement difficiles. C'est le cas de la Côte d'Ivoire qui va devoir en outre aborder, après la réussite de son offre en volume, l'étape difficile de la replantation d'un verger vieillissant. L'histoire de l'offre cacaoyère mondiale [17] montre que ce défi n'a généralement pas réussi à être surmonté par les différents pays producteurs. Aussi le pays doit-il aujourd'hui innover dans des politiques publiques rénovées, qui lui permettront de construire un environnement économique et politique incitatif, propice à l'investissement durable et à la diversification, et nécessaire à la préparation de l'après-cacao. Ces perspectives à terme laissent bien sûr entiers les enjeux du bras de fer qui se joue actuellement entre le gouvernement ivoirien et ses planteurs, d'une part, et entre les firmes mondiales et les bailleurs de fonds internationaux, d'autre part. Les leçons de l'histoire du marché devraient cependant inviter le gouvernement à faire preuve de réalisme, par une analyse des caractéristiques et des véritables rapports de force de la période, et à investir dans le renforcement des capacités des acteurs locaux (coopératives, entreprises, structures inter-professionnelles) qui constitue la condition sine qua non et 
la seule véritable alternative à la reconquête durable de la marge de manœuvre perdue par les opérateurs ivoiriens.

\section{REFERENCES}

1. LOSCH B (2000). La Côte d'Ivoire en quête d'un nouveau projet national. Politique Africaine, 78 : 5 25.

2. LOSCH B (2000). Coup de cacao en Côte d'Ivoire : économie politique d'une crise structurelle. Critique Internationale, $9: 6-14$.

3. LOSCH B (1999). Le complexe café-cacao de la Côte d'Ivoire. Une relecture de la trajectoire ivoirienne. Montpellier : Thèse de doctorat, université de Montpellier I ; 555 p. + annexes.

4. MCCALLA AF (1966). A duopoly model of world wheat pricing. J Farm Economics, 48 : 711-27.

5. BATES RH (1997). Open-economy politics. The political economy of the world coffee trade. Princeton, Chichester : Princeton University Press ; 225 p.

6. GOMBEAUD JL, MOUTOUT C, SMITH S (1999). La guerre du cacao. Histoire secrète d'un embargo. Paris : Calmann-Levy ; $218 \mathrm{p}$.

7. DAVIRON B (1993). Conflit et coopération sur le marché international du café. Une analyse de longue période. Montpellier : Thèse de doctorat, École nationale supérieure d'agronomie de Montpellier ; 299 p. + annexes.

8. OMAN C (1994). Globalisation et régionalisation : quels enjeux pour les pays en développement. Paris : OCDE ; $152 \mathrm{p}$.

9. MCMICHAEL P (1996). Development and social change. A global perspective. Thousand Oaks, London, New Delhi : Pine Forge Press ; 310 p.

10. VAN DER LAAN HL (1993). Boosting agricultural exports? A marketing channel perspective on an African dilemma. African Affairs, 92 : 173-201.

11. COUSSY J (1988). Déstabilisation des oligopoles internationaux ? Introduction. Économies et Sociétés, série $\mathrm{P}$ « Relations économiques internationales », 31 : 5-20.

12. DAVIRON B, LOSCH B (1997). Quelles stratégies sectorielles agricoles pour l'aide publique française ? Réflexions à partir des secteurs café-cacao africains. Paris : Notes et études $\mathrm{n}^{\circ} 60$, Caisse française de développement; $75 \mathrm{p}$.

13. MIGHELL R, JONES M (1963). Vertical co-ordination and contract farming. Washington : Report $\mathrm{n}^{\circ}$ 19, US Department of Agriculture.

14. JAFFEE S (1993). Exporting high-value food commodities. Success stories from developing countries. Washington : World Bank Discussion Papers $198 ; 105$ p.

15. BUREAU NATIONAL D'ÉTUDES TECHNIQUES ET DE DÉVELOPPEMENT (2000). Dispositif de suivi- 
évaluation de la libéralisation des filières café-cacao. Campagne de commercialisation 1999/2000. Abidjan : Observatoire café-cacao, Bnedt ; $18 \mathrm{p}$.

16. KIEFFER GA (2000). Cargill relance la guerre du cacao en Côte d'Ivoire. La Tribune, november 11.

17. RUF F (1995). Booms et crises du cacao. Les vertiges de l'or brun. Paris, Montpellier : ministère de la Coopération, Karthala, Cirad-Sar ; 459 p.

Illustrations

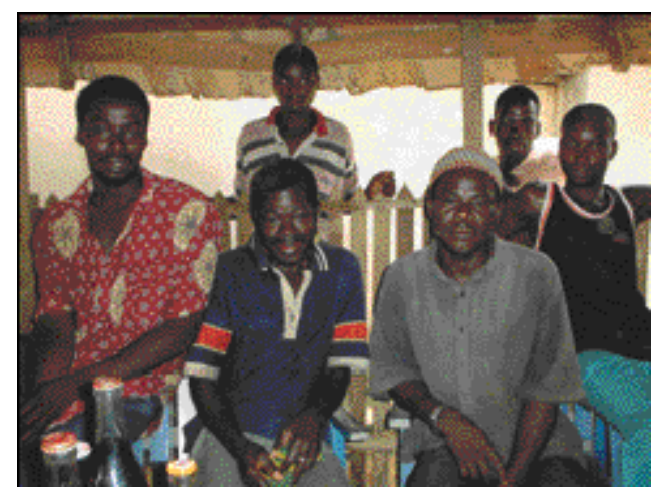

Photo. Migrants (crédit photo : F. Ruf).

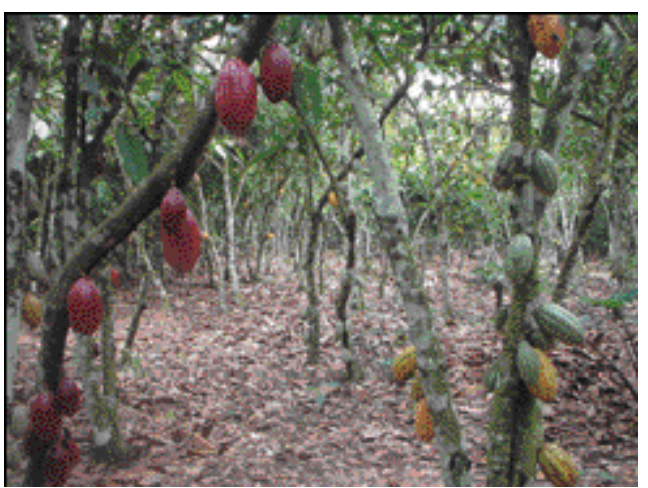

Photo. Cacaoyères (crédit photo : F. Ruf). 


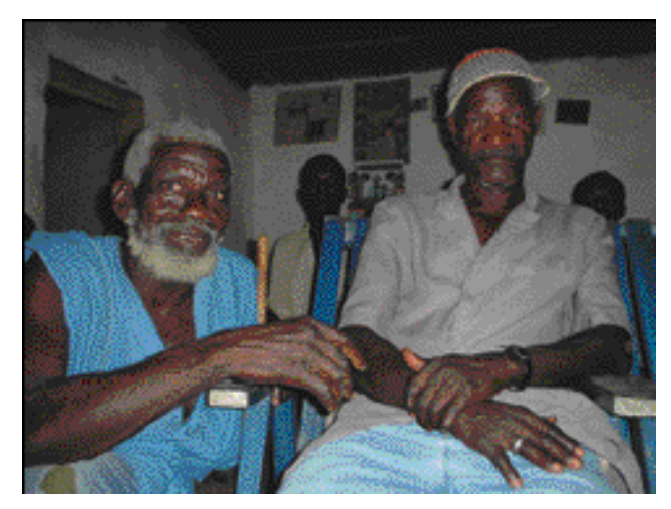

Photo. Vieux planteurs (crédit photo : F. Ruf).

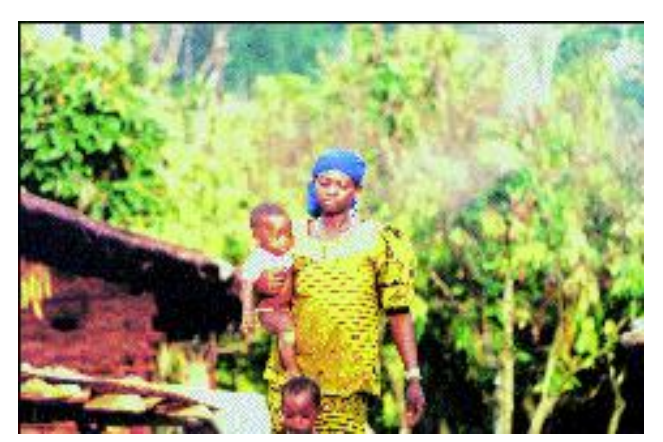

Photo. Une femme de planteur, sur fond de front pionnier, Sud-Ouest Côte d'Ivoire, à la lisière de la forêt de Taï (crédit photo : F. Ruf). 